tions led him to infer not only that synaptic contacts existed, but also that the unseen synaptic gaps were the basis of neurotransmission. With uncanny accuracy, he foretold today's hottest research: Cajal envisioned that tiny dendritic protuberances (spines) were important postsynaptic elements and that learning and memory might reflect the growth of new contacts. Rapport does well in making
Cajal accessible to non-neuroscientists. And for those of us who still ponder his drawings to guide us through the tangled forest, Rapport reminds us how lucky we are to have Cajal as our guide and companion.

Jeffry S. Isaacson is in the Department of

Neuroscience, University of California,

San Diego School of Medicine, La Jolla,

California 92093-0608, USA.

\title{
An ill-defined idea?
}

\section{Lovers and Livers: Disease Concepts in \\ History by Jacalyn Duffin \\ University of Toronto Press: 2005. 240 pp. $\$ 55, £ 35$ (hbk); \$27.50, £20 (pbk)}

\section{Andrew Scull}

Jacalyn Duffin's brief but beguiling book (it contains only 127 pages of text, the rest being consumed by notes and bibliography) is a revised version of the Joanne Goodman lectures, which she delivered at the University of Western Ontario in London, Canada, in 2002. Judging by the sprightliness of her prose, she must have provided an entertaining time for her audience. Along the way, her listeners will have encountered a clever series of arguments for viewing diseases as ideas, and a sometimes passionate dissection of disease and illness, doctor and patient, culture and pathology. At her best, Duffin creates a genuine sense of excitement and engagement with her materials, and these qualities are nowhere more evident than in her concluding chapter on livers (diseases thereof), where she draws fruitfully on her own clinical experience as a haematologist.

The notion that diseases may be thought of as "ideas influenced by the tastes and preoccupations of society" is likely at first to raise hackles in some quarters. Not another postmodernist rant, some will sigh, and sure enough, on the very first page there is a reference to the archfiend Michel Foucault. Not to worry, Duffin is not in the business of denying biological realities. Her point is far more clever and subtle, revolving around a distinction that she is scarcely the first to draw between disease and illness. This is, she concedes, a linguistic convention that will never be observed at the level of everyday speech, but making it here allows us to talk intelligently about two very different phenomena. Illness "applies to the subjective aspects of suffering, the problem experienced by the individual patients" and changes very slowly, if at all, over time; disease, in contrast, refers to "our ideas about that illness", ideas that not only describe symptoms and sufferers, but also incorporate an explanation or a theory about the illness. And diseases often change and proliferate as we recognize and add entities to the list of ailments that afflict the human race. It is this process of medicalization and demedicalization that Duffin's substantive chapters seek to illuminate.

Illnesses - that is, the symptoms experienced and reported by patients - often loom large in the making of a disease concept. Indeed, Duffin contends, "During early modern times, one could not be sick without feeling sick," and even today there are diseases still constructed solely on the basis of symptoms. The 'mental illnesses' that fill the pages of the American Psychiatric Association's Diagnostic and Statistical Manual of Mental Disorders are the most numerous, although scarcely the only, examples of the phenomenon. In the modern age, however, patients no longer have the final say on whether they are sick, as modern medicine tends to rely on the signs its techniques and technology can detect - it can even spot hypertension or latent cases of hepatitis $\mathrm{C}$ in asymptomatic people. Alternatively, unable to detect the biological underpinnings of conditions such as chronic fatigue syndrome, the profession uses its cultural authority to cast doubt on the reality of the complaint.

Love has symptoms too, for it has profound physical and psychological effects. Duffin points out that in an era of symptom-based medicine, 'lovesickness' emerged as a clinically respectable disease characterized by such symptoms as anorexia, insomnia and melancholy. It turns out to have an ancient pedigree and lingered in the repertoire of diseases until remarkably recently. Duffin even argues that love "still carries disease overtones in the medical and cultural psyche" today, something she attributes to the fact that it threatens to bring the "loss of control".

I found this portion of the book only intermittently compelling and occasionally selfindulgent. By contrast, Duffin's final chapter on the rise of hepatitis C fizzes with information and ideas that draw the reader into her argument. No one who reads this book will ever again think there is a 'routine' blood transfusion, for example, the hidden perils of this often life-saving procedure being starkly laid bare. More broadly, Duffin's examination of the proliferating array of hepatic diseases leads one to reflect upon a series of ironies surrounding the diagnosis and treatment of disease in the modern era, and to confront the continuing intrusion of moral values into the supposedly value-free realm of medicine.

Consider, for example, the construction of 'guilty' and 'innocent' victims of AIDS and hepatitis C; or the legal and ethical morass that surrounds the question of whether those infected by the blood supply should be financially compensated for their suffering, and by whom; or the complicated and to some degree perverse consequences for individual patients of the discovery that they have a symptomless, untreatable disease that may or may not lead to debility and death at some unknowable time in the future. Life lived beneath the sword of Damocles acquires a whole new meaning, and not one that most of us would welcome. Although as Duffin would be the first to point out, in reality we all exist in such a state anyway, and contrive to hide this painful truth from ourselves at all costs. Andrew Scull is in the Department of Sociology, University of California, San Diego, 9500 Gilman Drive, La Jolla, California 92093-0533, USA.
Call a doctor: when diseases were defined by symptoms alone, lovesickness was a clinically respectable diagnosis. 